\title{
Sistema de riego automatizado loT aplicado en el cultivo del pepino (Cucumis sativus)
}

\author{
Sanchez, Eval \\ Universidad Tecnológica Oteima \\ David, Panamá \\ eval.sanchez@oteima.ac.pa \\ Sanchez, Erick \\ Universidad Tecnológica Oteima \\ David, Panamá \\ tecnologia@oteima.ac.pa \\ Acosta, Aris \\ Universidad Tecnológica Oteima \\ David, Panamá \\ aacosta@oteima.ac.pa \\ González, Edmundo \\ Afiliación Institucional \\ David, Panamá \\ coordinadorinvestigacion@oteima.ac.pa
}

\section{Abstract}

An experiment was developed in which an automated loT irrigation system (T1) was implemented vs a manual irrigation (T2), nine cucumber plants were planted for each system in coconut fiber culture bags, for automated irrigation a drip irrigation mechanism and a tank was installed where water and nutrient solutions were added, manual irrigation was carried out with a container, a soil moisture and relative humidity sensor was installed, all data was stored in the application ThingsSpeak website to monitor substrate humidity and relative humidity, the cucumber harvest was carried out on the 64th day of plant development.

Keywords: Cucumber, Hydroponics, IoT, Irrigation, NodeMCU. 


\section{Resumen}

Se desarrollo un experimento en donde se implemento un sistema de riego automatizado loT (T1) vs un riego manual (T2), se sembraron nueve plantas de pepino por cada sistema en sacos de cultivo de fibra de coco, para el riego automatizado se incorporo un mecanismo de riego por goteo y se instalo un tanque donde se añadió el agua y las soluciones nutritivas, el riego manual se realizó con un recipiente, se instaló un sensor de humedad del suelo y de humedad relativa, todos los datos se almacenaron en la aplicación web de ThingsSpeak para monitorear la humedad del sustrato y la humedad relativa, la cosecha de los pepinos se realizo en el día 64 de desarrollo de la planta.

Palabras claves: Hidroponía, loT, NodeMCU, pepino, Cucumis sativus, riego.

\section{INTRODUCCIÓN}

Una gran ventaja del microcontrolador NodeMCU es que se puede conectar a internet mediante una conexión WiFi, esto permite enviar datos de sensores y almacenarlos para realizar distintos análisis, esto permite que se pueda desarrollar sistemas de riego inteligentes basados en el internet de las cosas y así se pueda monitorear lo que sucede en la finca [1].

El sensor de humedad del suelo permite tomar lecturas en tiempo real del sustrato en donde se encuentren sembrados los cultivos, este parámetro es considerado para la activación del riego a objeto de evitar que no se sature el sustrato, otro sensor importante es el de humedad relativa, este mide la cantidad de partículas de agua presentes en el ambiente, ambos parámetros son vitales para controlar el riego [2].

El cultivo hidropónico en fibra de coco permite una gran retención de humedad, acorde a las altas demandas de la planta de pepino, la fibra es excelente para la producción. En el agua de riego se añaden todas las soluciones nutritivas y se distribuye por un mecanismo de riego por goteo, de esta manera las plantas absorben mejor los nutrientes [3].

El principal objetivo de este sistema de riego automatizado es observar el rendimiento que tiene la producción de pepino referente a su cosecha haciendo uso de esta tecnología frente a otras soluciones de riego que existen actualmente. 


\section{MÉTODO}

\section{A. Riego Automatizado}

Se desarrollo e instalo un dispositivo para automatizar el riego, para esto se utilizó hardware basado en Arduino y el microcontrolador NodeMCU, se incorporó, además, un sensor de humedad del suelo y un sensor de humedad relativa y temperatura.

El riego se activa y se desactiva si se cumple con los parámetros de humedad del suelo y humedad relativa, para esto se desarrollo un algoritmo en lenguaje $C$ basado en la función condicional if-else, esto permite que se encienda una bomba de agua que esta conectada a un mecanismo de riego por goteo.

Mediante una conexión WiFi se envían todos los datos que generan los sensores a la nube de la aplicación web ThingSpeak, esto permite ver las lecturas de los sensores en tiempo real desde cualquier ubicación, para tener acceso a los datos en dispositivos móviles se utilizó la aplicación ThingView.

Se sembraron nueve plantas de pepino variedad Poinsett 76 en sacos de cultivo de fibra de coco DutchPlantin, se coloco una estaca de riego por goteo cerca del tallo de cada planta, esto con el fin de suministrar la solución nutritiva.

En un tanque de doscientos litros de capacidad se añadió dos tipos de soluciones nutritivas:

- Solución mayor A:

Esta solución contiene los macronutrientes como lo son el nitrógeno, fosforo, potasio, azufre y magnesio [4].

- Solución menor B:

La solución menor contiene los micronutrientes como el hierro, manganeso, cobre, zinc y boro.

Dentro del tanque se agregó cuarenta litros de agua, también se aplico dos tipos de dosis de solución nutritiva, del trasplante a la floración de la planta de pepino se añadía dos mililitros por litro de agua $(2 \mathrm{ml} / \mathrm{l})$ de solución mayor $A$ y un mililitro por litro de agua $(1 \mathrm{ml} / \mathrm{l})$ de solución menor $B$, de la floración a la cosecha la dosis se aumento a 4 mililitros de solución mayor $\mathrm{A}$ y dos mililitros de solución menor $\mathrm{B}$, cada vez que el tanque quedaba vacío se recargaba usado el mismo método.

Para registrar las lecturas permanentes de humedad del sustrato de fibra de coco, se enterró el sensor de humedad del suelo en un saco de cultivo cerca de la estaca de riego por goteo, el sensor de humedad relativa y temperatura se instalo dentro de la caja del dispositivo de riego.

\section{B. Riego Manual}

Se sembraron nueve plantas de pepino variedad Poinsett 76 en tres sacos de cultivo de 
fibra de coco, no se instaló ningún mecanismo de riego por goteo.

En un tanque de $20 \mathrm{~L}$ de capacidad se preparo una solución nutritiva usando la misma dosificación de solución mayor A y solución menor B que se empleo en el riego automatizado.

Para regar cada planta se uso $177 \mathrm{ml}$ de solución nutritiva dividida en dos tiempos de aplicaciones, el primero entre las 7:00 am y 10:00 am y el segundo entre las 3:00 pm y 6:00 pm, el riego se efectuó de forma manual usando un recipiente.

\section{RESULTADOS}

\section{A. Ensamblaje de ambos sistemas de riego}

A continuación, se presenta el ensamblaje del sistema de riego automatizado y el riego manual, el mecanismo de riego por goteo y los sacos de cultivo de fibra de coco, todo el experimento se desarrollo en un invernadero en donde se cubrió únicamente el techo.

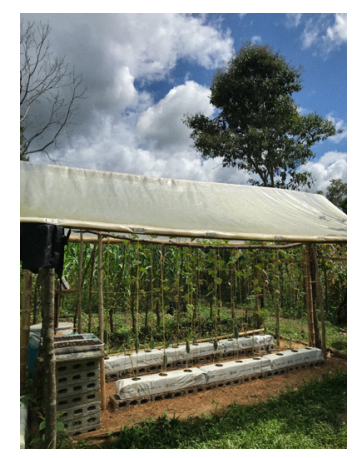

Fig. 1 Dispositivo de control de riego

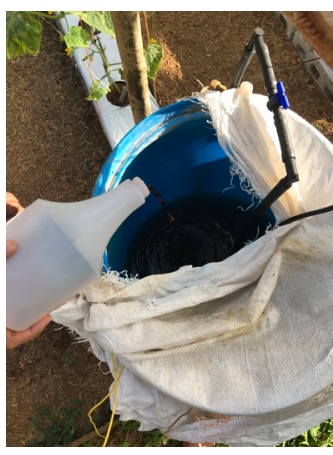

Fig. 2 Sensor de humedad en el sustrato

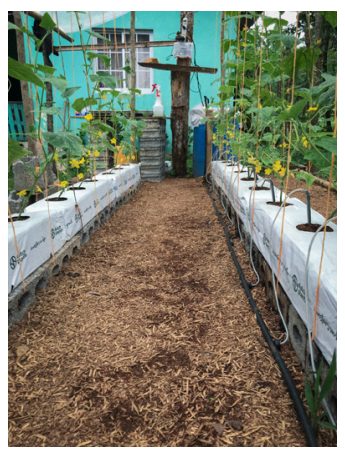

Fig. 3 Mecanismo de riego por goteo y riego manual

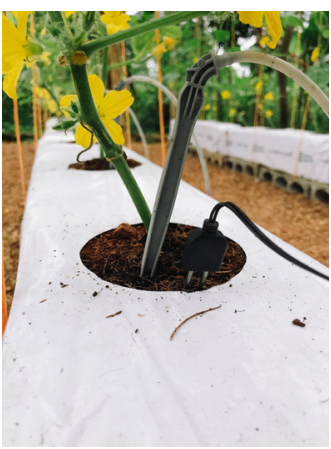

Fig. 4 Aplicación de soluciones nutritivas en el agua

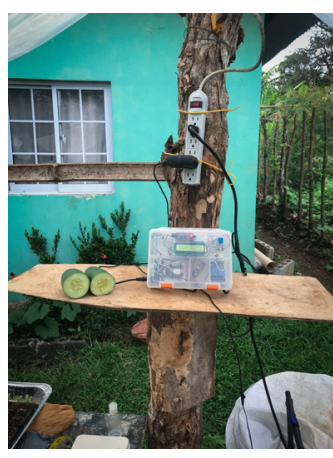

Fig. 5 Laboratorio de experimentación

El algoritmo que controla el riego funciona gracias a una estructura de control ifelse-if, fue escrito en lenguaje $C$, cuando el porcentaje de humedad del sustrato es menor e igual a $46 \%$ y la humedad relativa es menor e igual a $60 \%$ y mayor o igual a $55 \%$ entonces se activa la bomba de agua, cuando el porcentaje de humedad del sustrato es mayor e igual a $49 \%$ y mayor e igual a $61 \%$ se desactiva la bomba de agua, si el porcentaje de humedad del sustrato es mayor e igual a $70 \%$ entonces hay una saturación de humedad

\section{B. Cosecha de la producción en ambos sistemas de riego}

Se muestra el resultado de la cosecha usando el sistema de riego automatizado, en el cuadro se puede observar el peso por cada planta y un total de $3241 \mathrm{~g}$, una longitud 
promedio de $18,72 \mathrm{~cm}$ y un diámetro promedio de $17,57 \mathrm{~cm}$ correspondiente a una parcela de nueve plantas.

Tabla 1. Producción con riego automatizado

\begin{tabular}{llll}
\hline Planta & Peso $(\mathbf{g})$ & Longitud $(\mathbf{c m})$ & Diámetro $(\mathbf{c m})$ \\
\hline P1 & 385 & 20.5 & 18 \\
P2 & 374 & 21 & 18 \\
P3 & 344 & 17 & 18.5 \\
P4 & 543 & 17.5 & 16.7 \\
P5 & 234 & 16 & 16 \\
P6 & 351 & 20.5 & 17.5 \\
P7 & 396 & 21 & 18.4 \\
P8 & 326 & 19 & 17 \\
P9 & 288 & 16 & 18 \\
Total & 3241 & 18,72 & 17,57 \\
\hline
\end{tabular}

En este cuadro se muestran el resultado de la cosecha usando el riego manual, se obtuvo una cantidad total de $1656 \mathrm{~g}$ de pepino, $18,72 \mathrm{~cm}$ de longitud promedio y un diámetro de $17,57 \mathrm{~cm}$ que corresponden a una parcela de nueve plantas.

\begin{tabular}{llll}
\hline Planta & Peso $(\mathbf{g})$ & Longitud $(\mathbf{c m})$ & Diámetro $(\mathbf{c m})$ \\
\hline P1 & 213 & 16.5 & 17 \\
P2 & 124 & 12.5 & 14.2 \\
P3 & 163 & 15 & 15.5 \\
P4 & 187 & 13.5 & 17.5 \\
P5 & 250 & 16.5 & 17 \\
P6 & 193 & 16 & 16 \\
P7 & 149 & 14 & 15 \\
P8 & 201 & 12 & 12.3 \\
P9 & 176 & 13 & 18 \\
Total & 1656 & 14,33 & 15,83 \\
\hline
\end{tabular}

En estas figuras se puede observar los pepinos cosechados tanto en la parcela con el sistema de riego automatizado como en la de riego manual, del trasplante a la cosecha ambos grupos de plantas se cosecharon a los 64 días de sembradas.

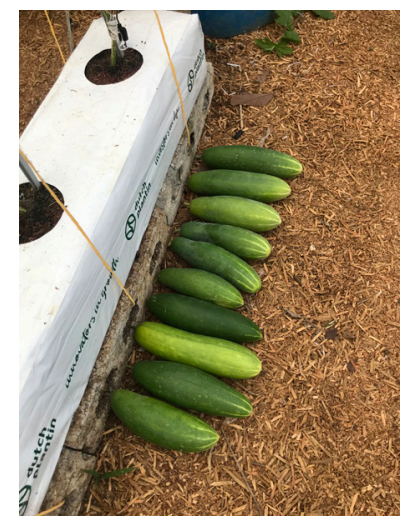

Fig. 6 Pepinos con riego automatizado

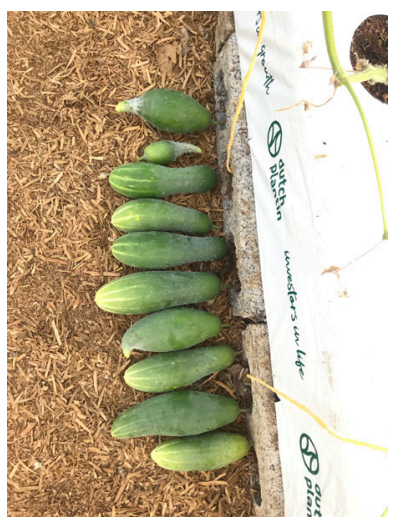

Fig. 7 Pepinos con riego manual 
De esta manera se muestran las graficas del porcentaje de humedad del sustrato, humedad relativa y temperatura, usando la aplicación web ThingsSpeak, en la base de datos se guarda cada lectura que generan los sensores y se puede acceder a la información en tiempo real.

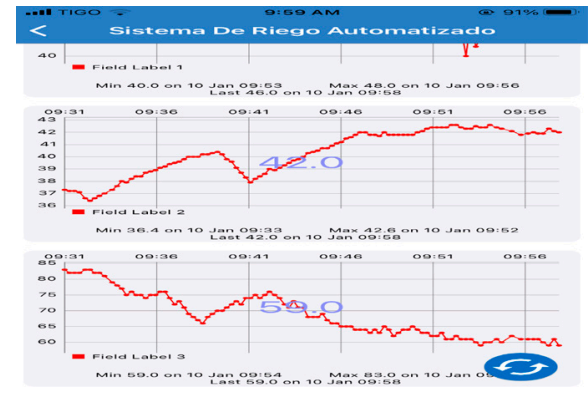

Fig. 8 Sensor de humedad del sustrato

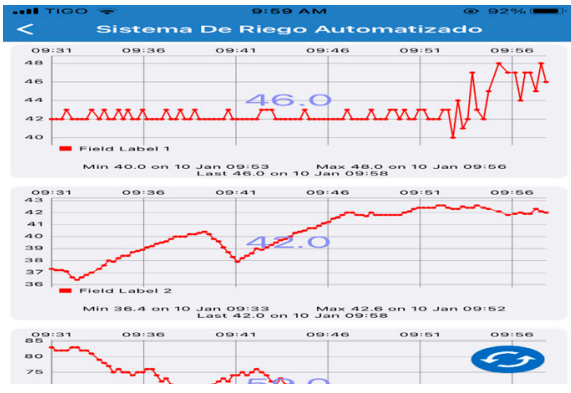

Fig. 9 Sensor de humedad relativa

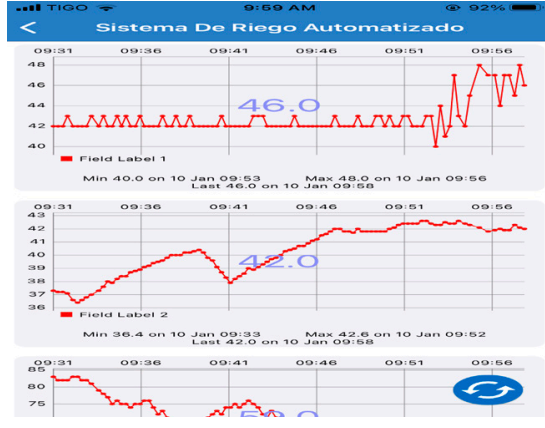

Fig. 10 Sensor de temperatura

\section{CONCLUSIONES}

Los pepinos cosechados haciendo uso del sistema de riego automatizado tienen una longitud más grande y diámetro aceptable para la variedad de pepino Poinsett 76, el tiempo de producción desde la siembra hasta la cosecha es de 65 días, en este grupo de plantas los pepinos ya estaban aptos para la cosecha y con un color verde oscuro muy aceptable para su comercialización, el sistema guardo todos los datos en la nube de ThingsSpeak y se puedo acceder a ellos en toda la etapa del desarrollo de las plantas; los pepino cosechados con el riego manual poseían menor tamaño y su forma no era cilíndrica, además la producción total fue menor que los pepinos de riego automatizado. El sistema es totalmente funcional por lo que se deben seguir realizado pruebas para mejorar todos los aspectos que surjan durante la experimentación.

\section{Referencias}

[1] A. R. Kholifah, K. I. Albar Sarosa, R. Fitriana, I. Rochmawati and M. Sarosa, "Drip Irrigation System Based on Internet of Things (IOT) using Solar Panel Energy," 2019 Fourth International Conference on Informatics and Computing (ICIC), 2019, pp. 1-6, doi: 10.1109/ICIC47613.2019.8985886.

[2] A. Hernan, G. Vargas, C.Ortiz and J.Vergara, "Diseño de un sistema de control y automatización de temperatura, humedad del suelo y humedad relativa para optimizar el rendimiento de cultivos bajo cubierta en CORHUILA", 2017: 4to Congreso Internacional AmITIC 2017, Aplicando nuevas tecnologías.

[3] P. Castro, J. Arontes, F.Contreras, Z. Rivera and Leova Martinez, "MANUAL DE PRODUCCION DE PEPINO EN BOLIS DE FIBRA DE COCO EN INVERNADERO", abril, 2018.

[4] F. Bravo, "Hidroponía familiar en sustrato: Hágalo fácil", Universidad de Costa Rica, 12 de noviembre del 2015. 


\section{Autorización y Licencia CC}

Los autores autorizan a APANAC XVIII a publicar el artículo en las actas de la conferencia en Acceso Abierto (Open Access) en diversos formatos digitales (PDF, HTML, EPUB) e integrarlos en diversas plataformas online como repositorios y bases de datos bajo la licencia CC:

Attribution-NonCommercial-ShareAlike 4.0 International (CC BY-NC-SA 4.0) https://creativecommons.org/

licenses/by-nc-sa/4.0/.

Ni APANAC XVIII ni los editores son responsables ni del contenido ni de las implicaciones de lo expresado en el artículo. 